\title{
Noise Property of a Quadrature Balanced VCO
}

\author{
Sangsoo Ko, Student Member, IEEE, and Songcheol Hong, Member, IEEE
}

\begin{abstract}
A quadrature balanced voltage controlled oscillator (B-VCO) with current source switching is proposed and analyzed. This letter shows analytically that the switching improves the phase noise. A switched transistor is also used as a coupling transistor to generate quadrature signals without degrading the phase noise. To investigate the effect of quadrature coupling on the phase noise, a single B-VCO and a quadrature B-VCO are implemented with identical components in an 0.18- $\mu \mathrm{m}$ CMOS process. Both VCO cores draw about $8.8 \mathrm{~mA}$ under a low bias voltage of $1.8 \mathrm{~V}$. The oscillation frequencies are $10.21 \mathrm{GHz}$ and $10.81 \mathrm{GHz}$. The measured phase noises of the single at an offset frequency of $1 \mathrm{MHz}$ VCO is $-114.83 \mathrm{dBc} / \mathrm{Hz}$ while that of the quadrature VCO is $-116.67 \mathrm{dBc} / \mathrm{Hz}$. The quadrature $\mathrm{B}-\mathrm{VCO}$ is superior to the single $\mathrm{B}-\mathrm{VCO}$ with respect to phase noise and oscillation frequency in the X-band.
\end{abstract}

Index Terms-Balanced, CMOS, current switching, noise, quadrature, transformer, voltage controlled oscillator (VCO).

\section{INTRODUCTION}

$\mathbf{T}$ HE quadrature signal source is a key component in imagerejection transceivers. Recently, the many different topologies of a differential (cross-coupled) voltage controlled oscillator (VCO) have enabled many quadrature VCOs to be implemented because of the ease with which a quadrature coupling transistor can be added [1], [2].

A CMOS differential VCO with a p-MOS and an n-MOS is superior at low frequency. However, a VCO in the $\mathrm{x}$-band is composed of only an n-MOS since a p-MOS is unavailable at about the $\mathrm{x}$-band. The noise property of a differential VCO with only an n-MOS is inferior to that of a B-VCO in a high frequency VCO [3]. Nonetheless, a B-VCO consumes a high bias current to have a reliable start-up. To overcome the disadvantages of a B-VCO, current source switching is used [4]. However, the topology also affects the superior phase noise property of a B-VCO because the topology is similar to a differential VCO This letter shows that the switching analytically improves the phase noise and that the $\mathrm{B}-\mathrm{VCO}$ is proposed to generate quadrature signals in the $\mathrm{X}$-band.

\section{Circuit Design}

The proposed B-VCO block with current source switching consists of n-MOS transistors and a transformer composed of $L_{1}$ and $L_{2}$ as shown in Fig. 1. The capacitive voltage divider that provides a positive feedback path is formed by $C_{f 1}$ and $C_{f 2}$.

Manuscript received August 18, 2004; revised March 21, 2005. This work was supported by the KOSEF-supported Millimeter-Wave Innovation Technology Research Center. This review of this letter was arranged by Associate Editor J.-J. Ma.

The authors are with the Department of Electrical Engineering and Computer Science, Korea Advanced Institute of Science and Technology (KAIST), Daejeon 305-701, Korea (e-mail: constant@eeinfo.kaist.ac.kr).

Digital Object Identifier 10.1109/LMWC.2005.856849

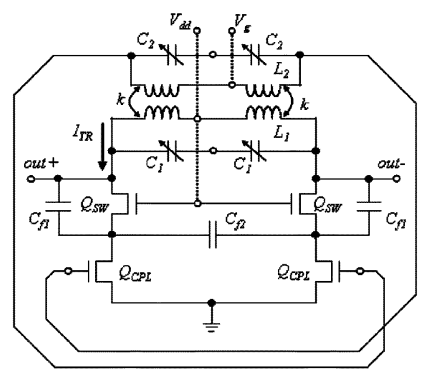

(a)

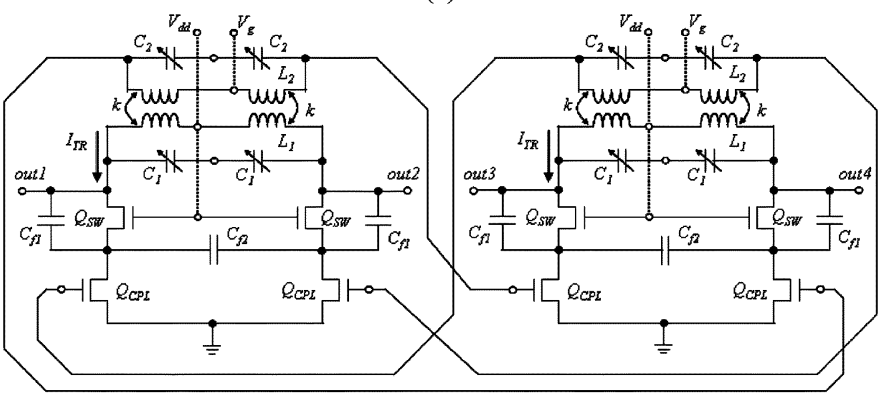

(b)

Fig. 1. Schematics of the proposed (a) single and (b) quadrature balanced VCOs.

In a conventional structure, $Q_{S W}$ and $Q_{C P L}$ are transistors for negative resistance and current source, respectively. However, a gate of $Q_{C P L}$ in the proposed structure is connected to the output of $L_{2}$ and the $C_{2}$ resonator for a current source switching. The $L_{2}$ of the transformer is connected to the external bias of $V_{g}$. Thus, the gate bias of $Q_{C P L}$ is controlled under a fixed supply voltage. It is useful to observe the effects of a bias current. The components of the VCOs that generate differential (out \pm ) and quadrature signals (out $1 \sim 4$ ) are identical. The two VCOs differ only in the connections of the feedback signal, as shown in Fig. 1 [5].

\section{NOISE ANALYSIS}

A balanced topology and a differential topology have been compared analytically [3]. The transistor in this letter is a BJT. However, this analysis is useful for a MOS because the dominant noise source is a channel noise. This letter shows that the negative conductance and noise properties in the $g_{m}$ cell of a balanced topology are superior to those of a differential topology at high frequency. However, a balanced topology with current switching differs from a conventional balanced topology in a phase noise property. The cross connected topology in current source switching is similar to the differential topology. In this section, we used analytic noise models to investigate the effect of the current switching.

A phase noise simulation includes harmonic balanced simulations with a nonlinear large-signal model. Although the model 


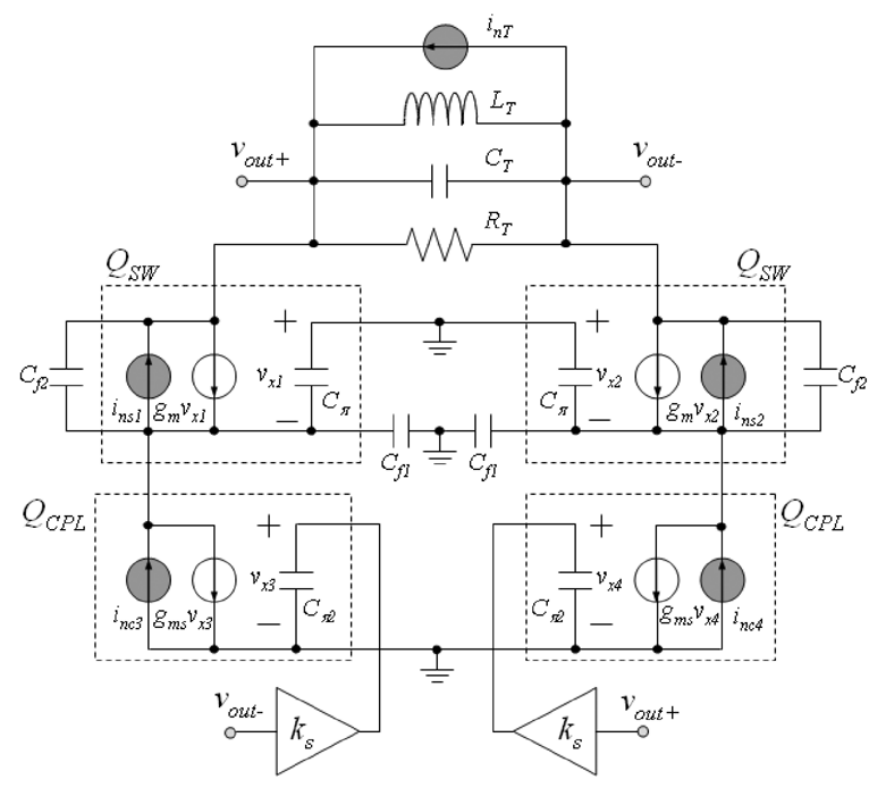

Fig. 2. Equivalent circuit of the single B-VCO with noise source.

is useful for predicting the accurate phase noise, this is too complex to help a chip-designer understand the VCO operations and phase noises. However, a linear phase noise model is simple and gives useful insight into the phase noises although the model is not accurate [3], [7].

Fig. 2 represents the linear models of single balanced topologies. The transistor model excludes the gate drain capacitance $C_{\mu}$ and the output resistor $r_{0}$ for simplicity. The LC thank is modeled with $L_{T}, C_{T}$, and $R_{T}$. The model includes a single noise source, $i_{n c}$ or $i_{n s}$, which is the sum of the channel and flicker noises in a transistor. It also includes the LC tank noise $i_{n T}$. This analysis is similar to that of [3]. The output amplitude $v_{o}$ of the LC tank is described as the superposition of all the noise sources.

The noise power of the output signal $\left(v_{o}=v_{\text {out }+}-v_{\text {out }-}\right)$ is represented by summing up all the noise powers as follows:

$$
\overline{v_{o}^{2}}=\frac{1}{2} \cdot\left(2 \cdot a_{S W}^{2} \cdot \overline{i_{n s}^{2}}+2 \cdot a_{C P L}^{2} \cdot \overline{i_{n c}^{2}}+2 \cdot a_{\mathrm{TANK}}^{2} \cdot \overline{i_{n T}^{2}}\right)
$$

where $a_{S W}, a_{C P L}$ and $a_{\mathrm{TANK}}$ indicate the noise contribution coefficients [3]. Each noise contribution coefficient is represented as follows:

$$
\begin{aligned}
a_{S W} & =\frac{s C_{f 1}}{2 P}+\frac{\left(\frac{s C_{f 1}}{2 P}-1\right)\left(s C_{f 1}+g_{m 1}\right)}{\frac{2 P}{s C_{f 1}} \cdot\left(s C_{\sigma}+g_{\sigma}\right)-\left(s C_{f 1}+g_{m 1}\right)} \\
a_{C P L} & =\frac{1}{s C_{f 1}} \frac{s C_{f 1}+g_{m 1}}{\frac{2 P}{s C_{f 1}} \cdot\left(s C_{\sigma}+g_{\sigma}\right)-\left(s C_{f 1}+g_{m 1}\right)}
\end{aligned}
$$

where

$$
\begin{aligned}
C_{\sigma} & =C_{f 1}+C_{f 2}+C_{\pi} \\
g_{\sigma} & =g_{m 1}+k_{s} \cdot g_{m 3} \\
P & =\frac{1}{s L_{T}}+\frac{1}{R_{T}}+s \cdot C_{T}+\frac{s \cdot C_{f 1}}{2}
\end{aligned}
$$

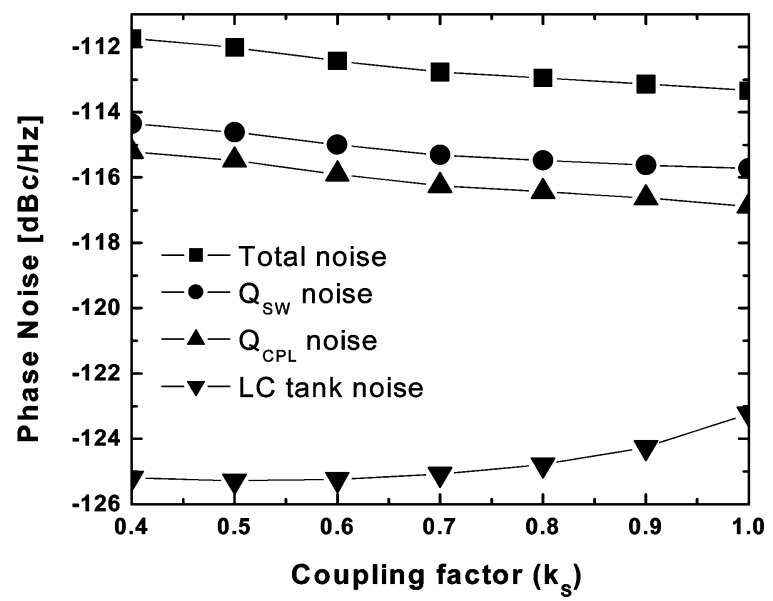

Fig. 3. Total phase noise at 1-MHz offset frequency and noise contribution of each component on a LC resonator.

and $k_{s}$ is the coupling coefficient. A conventional $\mathrm{B}-\mathrm{VCO}$ is $k_{s}=0$. If $k_{s}$ increases, the open loop gain and the effective trans-conductance $g_{\sigma}$ increase as follows:

$$
\begin{aligned}
\left|\frac{2 P}{s C_{f 1}}\right| & =\left|1+\frac{2}{s C_{f 1}}\left(\frac{1}{s L_{T}}+\frac{1}{R_{T}}+s C_{T}\right)\right| \\
& >1 \\
\left|\frac{2 P}{s C_{f 1}} \cdot\left(s C_{\sigma}+g_{\sigma}\right)\right| & >\left|s C_{\sigma}+g_{\sigma}\right| .
\end{aligned}
$$

Because of (5a) and (5b), when $k_{s}$ increases, $\left|a_{S W}\right|$ and $\left|a_{C P L}\right|$ decrease. In other word, $Q_{S W}$ and $Q_{C P L}$ contribute less noise to the LC tank. The current switching not only increases the open loop gain but also decreases the noise contribution of the active device noise

$$
a_{\mathrm{TANK}}=\frac{2}{2 \cdot P-s C_{f 1} \frac{s C_{f 1}+g_{m 1}}{s C_{\sigma}+g_{\sigma}}} .
$$

As shown in (6), the noise contribution of the tank $a_{\text {TANK }}$ increases as $k_{s}$ increases, whereas $\left|a_{S W}\right|$ and $\left|a_{C P L}\right|$ decrease. The parameter $\left|a_{\text {TANK }}\right|$ is not dominant in the phase noise. Thus, the total noise power decreases as $k_{s}$ increases.

This analysis is supported by the harmonic balanced simulation in the Agilent ADS with BSIM3 MOS model, which is shown in Fig. 3. The model shows the phase noise at a $1 \mathrm{MHz}$ offset frequency of a 10-GHz oscillation frequency with respect to the coupling factor $k_{s}$. The contribution of each noise source for $k_{s}$ agrees with the noise analysis. It shows that the noise of $Q_{C P L}$ is comparable to that of $Q_{S W}$ when the $\mathrm{LC}$ tank noise is quite low. According to some reports, the current switching of a B-VCO is used to decrease the bias currents and to ensure a reliable start-up [4]. We show, however, that current switching, which is readily achieved with quadrature coupling transistors, has the advantage of reducing noise from active devices. Moreover, the low noise property of proposed VCO is available when the topology is modified to generate quadrature signals [5].

\section{EXPERIMENTAL RESULTS}

The presented quadrature VCO is implemented in a standard $0.18 \mu \mathrm{m}$ CMOS process, which provided five layers of $\mathrm{Al}$ 


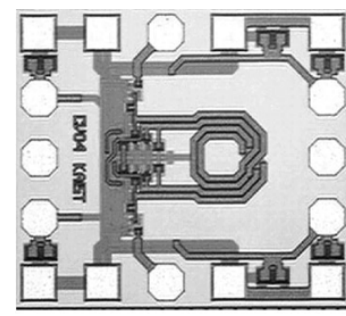

(a)

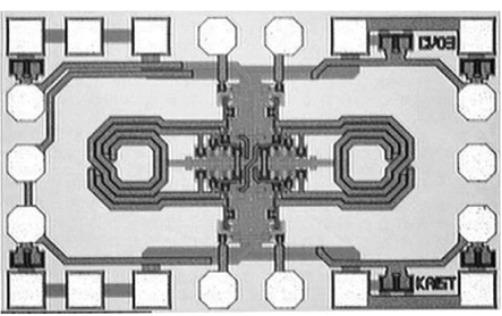

(b)
Fig. 4. Photographs of the fabricated (a) single $\left(670 \times 770 \mu \mathrm{m}^{2}\right)$ and (b) quadrature balanced VCO $\left(680 \times 1100 \mu \mathrm{m}^{2}\right)$.

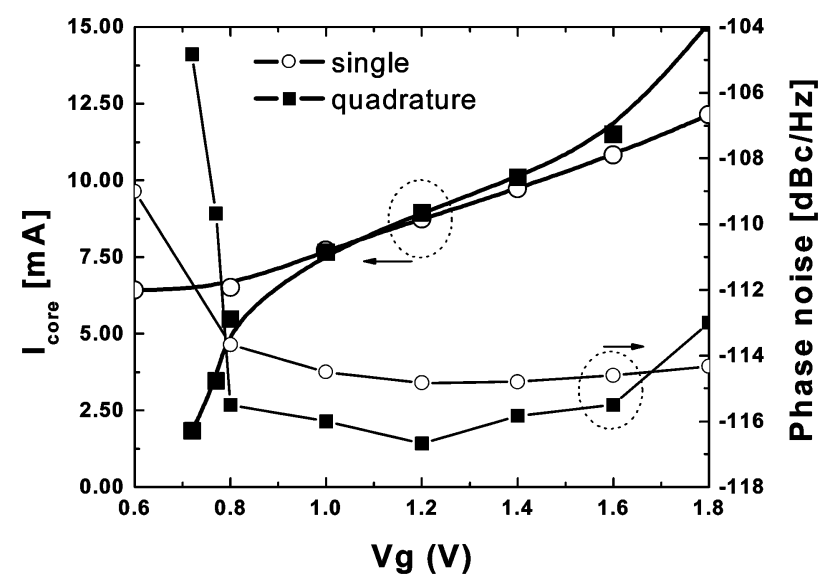

Fig. 5. Core bias currents and phase noise measurements at 1-MHz offset of single and quadrature $\mathrm{B}-\mathrm{VCO}$ as bias control voltage $\mathrm{Vg}$.

metal and 2- $\mu$ m-thick analog AlCu metal. As shown in Fig. 4, the chip sizes of the single $\mathrm{VCO}$ and the quadrature $\mathrm{VCO}$ were $670 \times 770 \mu \mathrm{m}^{2}$ and $680 \times 1100 \mu \mathrm{m}^{2}$. For a comparison of phase noises, all the components in both VCOs were identical.

The VCOs are measured by on-wafer probing with Agilent HP9764E spectrum analyzer and its accompanying kit for measuring phase noises. The VCO works under $1.8 \mathrm{~V}$ supply voltage. The cable loss in the measurement setup was about $2 \mathrm{~dB}$ at $10 \mathrm{GHz}$.

The oscillation frequency of the single VCO was $10.21 \mathrm{GHz}$, while that of the quadrature VCO was $10.81 \mathrm{GHz}$. The phase noise of the single $\mathrm{B}-\mathrm{VCO}$ was $-114.83 \mathrm{dBc} / \mathrm{Hz}$ at a $1-\mathrm{MHz}$ offset frequency. Theoretically, the quadrature coupling improves the phase noise by about $3 \mathrm{~dB}$ [5]. The phase noise in the quadrature VCO improved by $1.84 \mathrm{~dB}$. Although the improvement is lower than $3 \mathrm{~dB}$, the noise property of the proposed single B-VCO is available in the quadrature. Both VCOs showed very high figure of merit (FOM) [6] of $183 \mathrm{~dB}$ and $185 \mathrm{~dB}$. These results show, for the first time, that the balanced structure generates quadrature signals without degrading the phase noise performance.

The phase noise at a 1-MHz offset frequency and the bias current for the oscillation condition were measured by adjusting $V_{g}$ as shown in Fig. 5. The current of the quadrature VCO increased faster than that of the single VCO at $V_{g}>1.2 \mathrm{~V}$. The phase difference between the gate and the drain of $Q_{C P L}$ was about $180^{\circ}$ in the single VCO. The difference was about $90^{\circ}$ in the quadrature VCO because the gate was driven by the quadra- ture signals. When $\mathrm{Vg}$ increases, these properties cause the $I_{S W}$ of the quadrature VCO to increase faster than $I_{S W}$ of the single VCO. A part of $I_{S W}$ should be stored in $C_{2}$ due to the phase difference between $I_{C P L}$ and $I_{S W}$. Thus, the difference has effect on the initial bias current for the reliable start-up. The single VCO can oscillate until $I_{\text {bias }}=6.5 \mathrm{~mA}$, and the quadrature VCO can oscillate until $I_{\text {bias }}=7.5 \mathrm{~mA}$. The single VCO is superior in the start-up condition. However, if the bias of VCO is decreased after the VCO oscillates, the quadrature B-VCO does under lower bias current than that of the single B-VCO as shown in Fig. 5.

The minimum phase noise is at $I_{\text {bias }}=8.8 \mathrm{~mA}$ in both VCOs. The phase noise was almost unchanged with respect to $\mathrm{Vg}$, though the bias current increased with Vg. Generally, the noise power decreases as the bias current increases. The increase in the bias current causes an increase in not only the noise power but also the $g_{m}$ of the $Q_{C P L}$. Thus, the phase noise is almost constant. Section III shows that the noise decreases as the $g_{m}$ increases.

\section{CONCLUSION}

We proposed and analyzed an X-band CMOS quadrature B-VCO. Single and quadrature B-VCOs use current switching for a reliable start-up, for reduction of active noises and for quadrature coupling. We showed analytically that current switching improves the phase noise. The phase noise of the single VCO was $-114.83 \mathrm{dBc} / \mathrm{Hz}$ at the offset frequency of $1 \mathrm{MHz}$ at $10.21 \mathrm{GHz}$; the corresponding value for the quadrature $\mathrm{VCO}$ was $-116.67 \mathrm{dBc} / \mathrm{Hz}$ at the offset frequency of $1 \mathrm{MHz}$ at $10.81 \mathrm{GHz}$. The VCO cores consume about $8.8 \mathrm{~mA}$ from a $1.8 \mathrm{~V}$ supply. The single $\mathrm{VCO}$ achieved an FOM of $183 \mathrm{~dB}$, and the quadrature VCO achieved an FOM of $185 \mathrm{~dB}$. Except for the start-up condition, the quadrature VCO is superior to the single $\mathrm{VCO}$ in the phase noise, the oscillation frequency, the tuning range, and the FOM.

\section{ACKNOWLEDGMENT}

The authors would like to thank the Staff of TELTRON for chip fabrication and support.

\section{REFERENCES}

[1] P. Andreani, A. Bonfanti, L. Romanò, and C. Samori, "Analysis and design of a 1.8-GHz CMOS LC quadrature VCO," IEEE J. Solid-State Circuits, vol. 37, no. 12, pp. 1737-1747, Dec. 2002.

[2] D. Baek, T. Song, E. Yoon, and S. Hong, "8-GHz CMOS quadrature VCO using Transformer-Based LC tank," IEEE Microw. Wireless Compon. Lett., vol. 13, no. 10, pp. 446-448, Oct. 2003.

[3] D. Baek, S. Ko, J. Kim, and S. Hong, "Ku-Band InGaP/GaAs HBT MMIC VCOs with balanced and differential topologies," IEEE Trans. Microw. Theory Tech., vol. 51, no. 4, pp. 1353-1359, Apr. 2003.

[4] R. Aparicio and A. Hajimiri, "A noise-shifting differential colpitts VCO," IEEE J. Solid-State Circuits, vol. 37, no. 12, pp. 1728-1736, Dec. 2002

[5] S. Ko, H. D. Lee, D.-W. Kang, and S. Hong, "An X-band CMOS quadrature balanced VCO," in IEEE MTT-S Int. Dig., 2004, pp. 127-272.

[6] E. Hegazi, H. Sjöland, and A. A. Abidi, "A filtering technique to lower LC oscillator phase noise," IEEE J. Solid-State Circuits, vol. 36, no. 12, pp. 1921-1930, Dec. 2001.

[7] B. Razavi, "A study of phase noise in CMOS oscillators," IEEE. J. SolidState Circuits, vol. 31, no. 3, pp. 331-343, Mar. 1996. 